\title{
Direct Involvement of Androgen Receptor in Oxytocin Gene Expression: Possible Relevance for Mood Disorders
}

\author{
Dan Dai', Qiao-Chu Li', Qiong-Bin Zhu', Shao-Hua Hu², Rawien Balesar ${ }^{3}$, Dick Swaab ${ }^{1,3}$ and Ai-Min Bao ${ }^{*, 1}$ \\ 'Department of Neurobiology; Key Laboratory of Medical Neurobiology of Ministry of Health of China; Zhejiang Province Key Laboratory of Mental \\ Disorder's Management, Zhejiang Province Key Laboratory of Neurobiology, Zhejiang University School of Medicine, Hangzhou, China; \\ ${ }^{2}$ Department of Mental Health, First Affiliated Hospital, Zhejiang University School of Medicine, Zhejiang Province Key Laboratory of Mental \\ Disorder's Management, Hangzhou, China; ${ }^{3}$ Netherlands Institute for Neuroscience, an Institute of the Royal Netherlands Academy of Arts and \\ Sciences, Amsterdam, The Netherlands
}

\begin{abstract}
Oxytocin (OXT), synthesized in the hypothalamic paraventricular nucleus (PVN) and then released into different brain areas, may play a crucial role in various behaviors and neuropsychiatric disorders, including depression. Testosterone has been proposed by clinical studies to have the opposite effect of oxytocin in these disorders. We began by studying, in the postmortem hypothalamus of fifteen patients with mood disorders and fifteen matched controls, the expression of OXT in the PVN by means of immunocytochemistry (ICC) and the co-localization of OXT and androgen receptor (AR) by means of double labeling ICC. Subsequently, the regulatory effect of AR on OXT gene expression was studied in vitro. We found a higher expression of PVN OXT in the mood disorder patients than in the control subjects, and observed a clear co-localization of AR in OXT-expressing neurons, both in the cytoplasm and in the nucleus. In addition, a significant decrease in OXT-mRNA levels was observed after pre-incubation of the SK-N-SH cells with testosterone. A further potential androgen-responsive element in the human OXT gene promotor was revealed by electrophoretic mobility shift assays and cotransfections in neuroblastoma cells. Finally, in vitro studies demonstrated that AR mediated the down-regulation of OXT gene expression. These results suggest that the fact that OXT and testosterone appear to have opposite effects in neuropsychiatric disorders might be based upon a direct inhibition of AR on OXT transcription, which may provide a novel target for therapeutic strategies in depression. Neuropsychopharmacology (2017) 42, 2064-207I; doi:I0.1038/npp.2017.76; published online 31 May 2017
\end{abstract}

\section{INTRODUCTION}

The neuropeptide oxytocin (OXT) is synthesized in the hypothalamic paraventricular nucleus (PVN) and supraoptic nucleus (SON). When released from the neurohypophysis it may act as a neurohormone and it thus plays a role in uterine contractions and milk ejection during parturition and lactation (Carson et al, 2013). In recent decades, however, PVN OXT being synaptically released into different brain areas and playing a central role as a neuromodulator, has drawn increasing attention (Knobloch et al, 2012). Central effects of OXT include affective social behaviors, such as maternal care and pair bonding (Smith et al, 2010), the attenuation of fear and of the stress response (Quirin et al, 2011), and the modulation of the symptoms of psychiatric disorders such as major depressive disorder (MDD) and bipolar disorder (BD) (Cochran et al, 2013). The observations of the social and/or clinical effects of OXT have been

*Correspondence: Professor A-M Bao, Department of Neurobiology, Key Laboratory of Medical Neurobiology of Ministry of Health of China, Zhejiang Province Key Laboratory of Neurobiology, Zhejiang University School of Medicine, Hangzhou 3 1 0058, China, Tel: +86 57| 88208789 , Fax: +86 57I 88208640, E-mail: baoaimin@zju.edu.cn

Received 6 October 20 16; revised 5 April 2017; accepted 6 April 20 17; accepted article preview online 27 April 2017 generally performed by intranasal OXT administration. However, the belief that all effects of intranasal OXT can be explained by a direct OXT action on brain systems has been challenged (Leng and Ludwig, 2016). In addition, the literature tends to remain equivocal on the direction of OXT changes in depression. For instance, both enhanced (Heinrichs and Domes, 2008; Turan et al, 2013) and weakened (Ozsoy et al, 2009) serum OXT levels have been reported in mood disorders. These considerations render it crucial to do postmortem brain research on the OXT system in clinically and neuropathologically well-characterized patients with mood disorders. Although we had observed increased numbers of OXT-expressing neurons in the hypothalamic PVN in depression in a previous relatively small study (Purba et al, 1996), we needed to confirm this observation in a larger and independent brain sample, and we had to subsequently elucidate the mechanism of how OXT expression is regulated by testosterone.

A number of studies have pointed to the involvement of the steroid receptor family in the regulation of the activity of stress-related neuropeptide neurons in the hypothalamic PVN (Bao et al, 2006; Murakami et al, 2011; Watts, 2005), which might play an important role in the pathogenesis of mood disorders. Indeed, the human OXT promoter was found to be up-regulated by estrogens, when ER was present 
(Richard and Zingg, 1990). However, although a study by Zhou et al showed that OXT-expressing neurons in a particular part of the rat PVN, ie the ventral zone of the medial parvocellular part of the PVN, co-express androgen receptor (AR) (Zhou et al, 1994), an other study claimed that rat PVN lacked AR (Bingaman et al, 1994). In addition, we found clear AR expression in the human PVN (Bao et al, 2006; Fernándezguasti et al, 2000). It was recently proposed that there were opposing effects of OXT and testosterone in cognitive and behavioral functions and in various neuropsychiatric disorders, such as autism, schizophrenia and depression(Crespi, 2015), which makes the topic of a potential role of androgens in the regulation of OXT expression in human even more intriguing.

In the present study we therefore investigated, first in postmortem human brain tissue, the PVN OXT expression in depression and the possible presence of co-localization of the AR in OXT-immunoreactive neurons in the PVN. After we were able to confirm, in the largest sample to date, the presence of a larger amount of PVN OXT expression in depression patients and observed a clear co-localization of nuclear AR in OXT-immunoreactive neurons, we investigated the regulation of testosterone on OXT gene expression via $A R$ as a nuclear transcriptional factor, and subsequently observed an inhibiting effect of AR on human OXT gene expression.

\section{MATERIALS AND METHODS}

\section{Part 1: Immunohistochemistry in Human Hypothalamus}

Postmortem brain material was obtained from the Netherlands Brain Bank (director Dr Inge Huitinga) following permission for a brain autopsy and the use of the brain material and clinical data for research purposes. The Diagnostic and Statistical Manual of Mental Disorders (DSM)-III-R/DSM-IV) criteria were used for the diagnosis of MDD or BD at any time during life. In total, hypothalamic material of 15 mood disorder patients (nine MDD, six BD) and fifteen matched control subjects was studied. The absence of neuropathological changes in all these subjects was confirmed by systematic neuropathology (van de Nes et al, 1998). Detailed clinico-pathological information and $P$-values of the matched parameter are given in Supplementary Table S1.

The hypothalami were dissected at autopsy and fixed in $0.1 \mathrm{M}$ phosphate buffered $4 \% \mathrm{w} / \mathrm{v}$ formaldehyde ( $\mathrm{pH} 7.2)$ for 1-2 months. Tissues were dehydrated in graded ethanol, embedded in paraffin and serially cut into sections $(6 \mu \mathrm{m})$ on a Leitz microtome and stored at room temperature. To localize the borders of the PVN, thionine staining was performed on every 100th section throughout the hypothalamus. The rostral and caudal borders of the OXT-PVN were later determined by immunocytochemical staining of OXT in every 100th section, and the beginning and end of the OXT- PVN were defined as the sections where no OXT cells were present.

Immunocytochemistry of OXT-immunoreactivity in the hypothalamic PVN. Sections were incubated at $4{ }^{\circ} \mathrm{C}$ overnight with the OXT antibody $(1: 800$, OT-A-I-28, a kind gift from Dr F.W. van Leeuwen, who obtained it from Dr A. Hou
Yu). The next day, the sections were incubated at room temperature with a biotinylated anti-mouse antibody $(1: 400$, BA-1000, Vector Laboratories, USA) for $1 \mathrm{~h}$ and stained with $\mathrm{DAB}-\mathrm{Ni}$ substrate solutions (Sigma, Zwijndrecht, the Netherlands) for $15 \mathrm{~min}$.

Double-labeling of AR and OXT in hypothalamic PVN. For each subject, double staining of AR and OXT in hypothalamic PVN was performed on the section which contained the highest level of OXT-immunoreactivity. Sections were microwaved for $2 \times 5 \mathrm{~min}$ at $800 \mathrm{~W}$ in $0.05 \mathrm{M}$ Tris- $\mathrm{HCl}$ ( $\mathrm{pH} 9.0)$ to unmask antigens. The sections were incubated with the rabbit polyclonal AR antibody $(1: 800$, ab74272, Abcam, USA) for $1 \mathrm{~h}$, then kept at $4{ }^{\circ} \mathrm{C}$ overnight. The next day, after rinsing with TBS, the sections were incubated with mouse monoclonal OXT antibody $(1: 800$, OT-A-I-28, a kind gift from Dr F.W. van Leeuwen, who obtained it from Dr A. Hou Yu) for $1 \mathrm{~h}$. After subsequent rinsing with TBS, the sections were incubated with biotinylated anti-rabbit antibody $(1: 400$, BA-1000, Vector Laboratories) for $1 \mathrm{~h}$, followed by the incubation of $\mathrm{ABC}$ solution for $1 \mathrm{~h}$. After another rinse with TBS, the sections were stained red with Vector NovaRED Substrate Kit (SK-4800, Vector, USA) for $15 \mathrm{~min}$. After another rinse with TBS, the sections were incubated with alkaline phosphatase anti-mouse antibody $(1: 200,115-055-003$, Jackson ImmunoResearch, USA), and stained blue with Vector Blue Substrate Kit (SK-5300, Vector, USA) for $25 \mathrm{~min}$. Sections were then rinsed and cover-slipped using Kaiser's glycerin (Merck Chemicals, Germany).

Image analysis. Quantification of the integrated optical density (IOD) of the OXT-immunoreactive signal was performed as described in our previous study (Gao et al, 2012) with a minor modification, which can be found in Supplementary Materials and Methods. The total IOD of oxytocin-immunoreactivity as determined by computerassisted morphometry is not only an objective method but also less time-consuming than neuron-counting. In addition, the IOD shows a good positive correlation with neuroncounting, performed in the way we described previously (Fronczek et al, 2005): for twenty-two sections of one patient and one control: rho $=0.799, p<0.001$.

\section{Part 2: In Vitro Studies in Cell Lines}

Immunofluorescence. Human neuroblastoma SK-N-SH cells (Bioleaf Company, China) were cultured according to an existing protocol (Smets et al, 1989). After having been grown in 96-well plates for $48 \mathrm{~h}$, SK-N-SH cells were incubated with either polyclonal rabbit anti-AR antibody $(1: 50$, ab74272) or polyclonal rabbit anti-OXT antibody (ab2078, Abcam, USA) for $12 \mathrm{~h}$ at $4{ }^{\circ} \mathrm{C}$. After washing in PBS, the cells were incubated for $1 \mathrm{~h}$ with Alexa Fluor 488 goat anti-rabbit IgG $(1: 1000$, Beyotime Biotechnology, China) and mounted in FluorSave reagent (Beyotime Biotechnology, China). Images were captured with NIS-Elements imaging software (Nikon, Japan).

Effect of testosterone on OXT-mRNA expression. Testosterone (T1500, Sigma, USA) was diluted in $100 \%$ chloroform 
and used at a final concentration of $10^{-5} \mathrm{M}$ in $0.001 \%$ chloroform. Estrogen receptor (ER) antagonist ICI 182780 (1286650, Sigma, USA) was diluted in 100\% DMSO and used at a final concentration of $10^{-7} \mathrm{M}$ in $0.001 \%$ DMSO. SK-N$\mathrm{SH}$ cells were grown in 6-well plates for $12 \mathrm{~h}$ until they were attached to the bottom. Media containing FBS were then replaced by charcoal stripped FBS (Hyclone, USA). Once grown to $70 \%$ confluency, the SK-N-SH cells were exposed to ICI 182780 for $0.5 \mathrm{~h}$, followed by the addition of $10^{-5} \mathrm{nM}$ testosterone (final concentration in $2 \mathrm{ml}$ cell media) for $24 \mathrm{~h}$. Control cells were treated with the same content of chloroform and DMSO. Total RNA isolation and cDNA synthesis were performed as described earlier (Wang et al, 2008). QPCR procedures are indicated in Supplementary Materials and Methods.

Electrophoretic mobility shift assay (EMSA). In order to determine whether the human OXT promoter region contains potential androgen responsive elements (AREs), the human OXT gene promoter region was scanned with AR matrix models in an online database (http://jaspar.genereg. net) region (the nucleotide sequences of $O X T$ were provided by the National Center for Biotechnology Information database at http://www.ncbi.nlm.nih.gov/gene/5020). At position $-346 \mathrm{bp}$ and $+204 \mathrm{bp}$ of the human OXT gene promoter, there were two sequences (called site $A$ and site $B$, respectively in the present study) closely resembling an ARE (site A resemblance rate: $81.6 \%$; site $\mathrm{B}$ resemblance rate: 82.3\%; Figure 4a). Probes were designed based on the two candidate AREs. Sequences are shown in Supplementary Table S3. Nuclear extracts were made from 293T cells transiently transfected with PSV-AR. After transfection, nuclear proteins were extracted and identified by western blot. The experimental procedures are indicated in Supplementary Materials and Methods.

OXT luciferase reporter plasmid construction. To generate the reporter plasmids, two fragments from the human OXT gene promoter sequence were amplified from genomic DNA from Hela cells by nested PCR:

- a full-length fragment (from - $533 \mathrm{bp}$ to $+235 \mathrm{bp}$ ) denoted OXTA, which contained both potential ARE sites A and site $\mathrm{B}$.

- a shorter fragment (from -298 bp to $+235 \mathrm{bp}$ ) denoted OXTAm, which did not contain ARE site A.

For a schematic representation see Figure 5a. Fragments were amplified by nested PCR (see primers in Supplementary Table S4) and cloned into PGL3 luciferase reporter basic vector (E1751, Promega, USA).

OXT promoter fragments and PGL3 basic vectors were double-digested with restriction enzyme Kpn I and Hind III, ligated by $\mathrm{T} 4$ ligase, and amplified through bacterial transformation. The constructs were named as POXTA (containing OXTA) and POXTAm (containing OXTAm). The presence of the inserts was confirmed by DNA sequencing. The PRL-CMV renilla luciferase reporter construct (E2261, Promega, USA) was used as an internal control for plasmid transfection efficiency.
AR-OXT promoter interaction assay. 293FT cells were co-transfected at 70\% confluency with PSV-AR and with one of the OXT gene promoter plasmids (POXTA or POXTAm) and incubated for $24 \mathrm{~h}$ in media containing $10 \%$ charcoal stripped FBS. Subsequently, testosterone $\left(10^{-5} \mathrm{nM}\right)$ was added for $24 \mathrm{~h}$. Cells were fixed with $1 \%$ formaldehyde and stopped by glycine $(0.125 \mathrm{M})$.

Before proceeding to the immunoprecipitation, two validation experiments were performed to verify the successful transfection of PSV-AR and OXT gene promoter plasmids. First, a western blot was performed with the polyclonal AR antibody $(1: 200$, ab74272) and a secondary anti-rabbit horseradish peroxidase antibody (1:1000, MR-M100, MRBiotech, China). Secondly, PCR was performed using primers named Primer $\mathrm{A}$ and Primer $\mathrm{B}$, which were designed to amplify ARE site A and ARE site B respectively (primers are shown in Supplementary Table S5) for detection of ARE sites within POXTA and POXTAm transfected samples.

Next, immunoprecipitation was performed according to the instructions of the EZ-Magna ChIP A/G kit (17-10086, Millipore). This involved incubation of the POXTA- and POXTAm-transfected isolates with $5 \mu \mathrm{g}$ rabbit anti-AR polyclonal antibodies. As a specificity control, POXTA samples were also reacted with $1 \mu \mathrm{g}$ rabbit IgG. Untransfected cells were collected to serve as negative controls. Immunoprecipitated DNA was amplified by PCR using Primer A and primer B, respectively. Amplification products were analyzed by $2 \%$ agarose gel electrophoresis.

Luciferase reporter assay using the human OXT promoter. Since both EMSA and the AR-OXT promoter interaction assay showed that site A was the ARE on the OXT promoter, we investigated, by means of luciferase reporter assay, the effect of testosterone combined with AR on the OXT promoter. Co-transfection of 293 FT cells was performed using lipo 2000 (11668-027, Invitrogen). Cells were incubated with transfection reagent and plasmids PSV-AR and POXTA, together with the pRL-CMV vector (E2261, Promega, USA) as the internal control for $24 \mathrm{~h}$, followed by testosterone treatment $\left(10^{-5} \mathrm{nM}\right)$ in DMEM, which contained 10\% Charcoal Stripped FBS (Hangzhou Sijiqing biotech, China) to ensure steroid-free culture conditions. Twenty-four hours after the treatments, luciferase activity was measured using the dual-luciferase assay according to the manufacturer's protocol (E2920, Promega, USA). Relative light units were measured using the Varioskan Flash Multimode Reader (5250040, Thermo).

Statistical analysis. Data were analyzed using SPSS software (version 23). A Shapiro-Wilk normality test confirmed that the data were normally distributed. Student's $t$-test was applied to compare the differences between the experimental group and the control group. Also, a multiple linear regression model was applied to verify whether PVN OXTimmunoreactivity was associated with mood disorders, taking into consideration confounding factors such as sex, age, or postmortem delay. The Mardia-Watson-Wheeler test (Watson, 1982) was used for group comparisons of circular data, including the clock time of death and the month of death. $P<0.05$ was considered to be significant. 
Specificity of antibodies. Specificity of antibodies is indicated in Supplementary Materials and Methods.

\section{RESULTS}

\section{Mood Disorder Patients had Significantly Higher PVN OXT-Immunoreactivity}

The mood disorder group showed a larger amount of (44.1\%) PVN OXT-immunoreactivity than the control group $(p=0.027$, Figure 1$)$. In addition, multiple linear regression analysis revealed that PVN OXT-immunoreactivity was significantly associated with mood disorder $(\beta=6.798$, $p=0.039$ ), while sex, age, postmortem delay, fixation time and brain weight did not show a significant effect on PVN OXT-immunoreactivity $(p \geqslant 0.157)$. There was no significant difference in OXT-immunoreactivity between the MDD and the BD group $(p=0.584$, Figure 1). It should be noted, however, that some confounding factors, such as sex ratio, were not well-matched for the subgroup comparison.

\section{Co-Localization of AR and OXT-Expressing Neurons in the PVN}

OXT-immunoreactivity in the hypothalamic PVN was only present in the cytoplasm and in fibers, while AR-immunoreactivity was found in the nuclei, cytoplasm and fibers of OXT-expressing neurons. Some OXT-immunoreactive neurons (blue cytoplasm) were clearly co-expressing nuclear AR-immunoreactivity (red) (Figure 2).

\section{Testosterone Decreased OXT-mRNA In Vitro}

SK-N-SH cells showed clear endogenously expressed ARimmunoreactivity and OXT-immunoreactivity (Figure 3a). Significantly decreased OXT-mRNA levels were found after pre-incubation of the cells with the ER antagonist ICI 182780 followed by testosterone (student's $t$-test, $p=0.05$, Figure $3 \mathrm{~b}$ ).

\section{Identification of Potential Functional AREs in Human OXT Promotor}

In the EMSA study, only probe A (site A) strongly bound to nuclear extracts from 293T cells transfected with PSV-AR, shown as the two shifted bands on top of the gel (Figure 4b), which according to a previous study (Wilson and McPhaul, 1996) are AR variants of about 87 and $110 \mathrm{kd}$, respectively. A 200 -fold excess of unlabeled probe A was sufficient to outcompete the specific interaction with labeled probe $\mathrm{A}$, eliminating the shifted bands (Figure 4b). Altogether with previous findings that these two bands may combine with other nuclear compounds acting as AR co-regulator (Bennett et al, 2010), our data indicated that site A probably represents a functional ARE.

Co-transfection of 293FT cells with the AR-expression plasmids POXTA and POXTAm was proved by western blot detection of AR (Figure 5b, panel I) and PCR amplification of ARE sites A and B using Primer A and primer B respectively (Figure $5 b$, panel II).

The enrichment of AR-binding site A was confirmed in the POXTA co-transfected cell lysates with the anti-AR antibody, followed by PCR amplification of Primer A, while

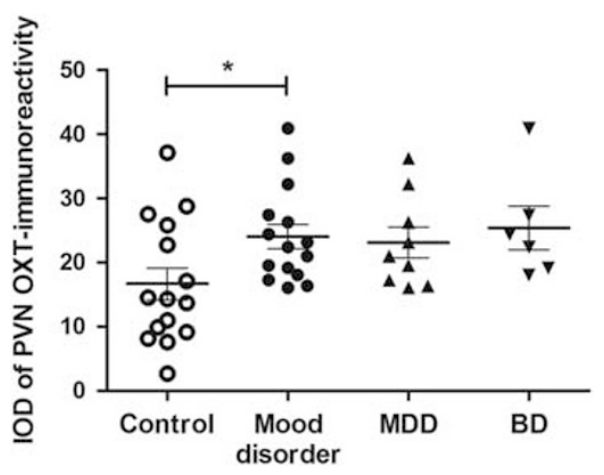

Figure I Comparison of oxytocin (OXT)-immunoreactivity in hypothalamic paraventricular nucleus (PVN) between mood disorder and controls. Data are presented as mean \pm SEM. BD: bipolar disorder; IOD: integrated optical density; MDD: major depressive disorder. ${ }^{*} p=0.027$.

the same isolates with nonspecific rabbit IgG failed to show the PCR product, as did the untransfected 293FT cell lysates with the same anti-AR antibody (Figure $5 b$ ). Furthermore, POXTAm co-transfected cell lysates with the anti-AR antibody did not demonstrate PCR product when Primer B was used, which indicated that site B does not interact with $\mathrm{AR}$ in the OXT promoter region. Together, these observations showed that AR binds only to ARE site A.

\section{AR Represses the OXT Promoter}

To examine how androgens might modulate OXT gene expression through the potential ARE (site A), the luciferase reporter plasmid POXTA was transiently transfected into 293FT cells, with or without co-transfection of the human AR expression vector PSV-AR, followed by testosterone treatment. OXT promoter transcriptional luciferase activity presented a significant decrease when, compared to controls without PSV-AR, cells were co-transfected with PSV-AR. This procedure was followed by testosterone treatment $(p=0.0126$, Figure 5c). This led us to conclude that testosterone may inhibit OXT gene expression, via an interaction between AR and ARE.

\section{DISCUSSION}

The data from our largest series of postmortem material so far confirm the presence of more PVN OXT expression in mood disorder patients (Purba et al, 1996), while the co-localization of AR in some of the human PVN OXT-immunoreactive neurons is a novel finding. The co-localization of AR, both in the cytoplasm and in the nucleus, in human PVN OXT neurons points to the possibility of a direct regulating role of androgens on PVN OXT gene expression.

OXT may contribute to symptoms of depression in different ways. OXT is a satiety hormone and the enhanced hypothalamic OXT levels we observed may thus be related to the eating disorders that often accompany depression (Baxter, 2016; Lawson et al, 2012; Wade et al, 2000). On the other hand, OXT has generally been associated with enhanced pro-social behaviors and the administration of OXT was found to reduce the symptoms of depression and anxiety (Hofmann et al, 2015). At first sight, our findings of a larger amount of PVN OXT-immunoreactivity and the elevated peripheral OXT levels 

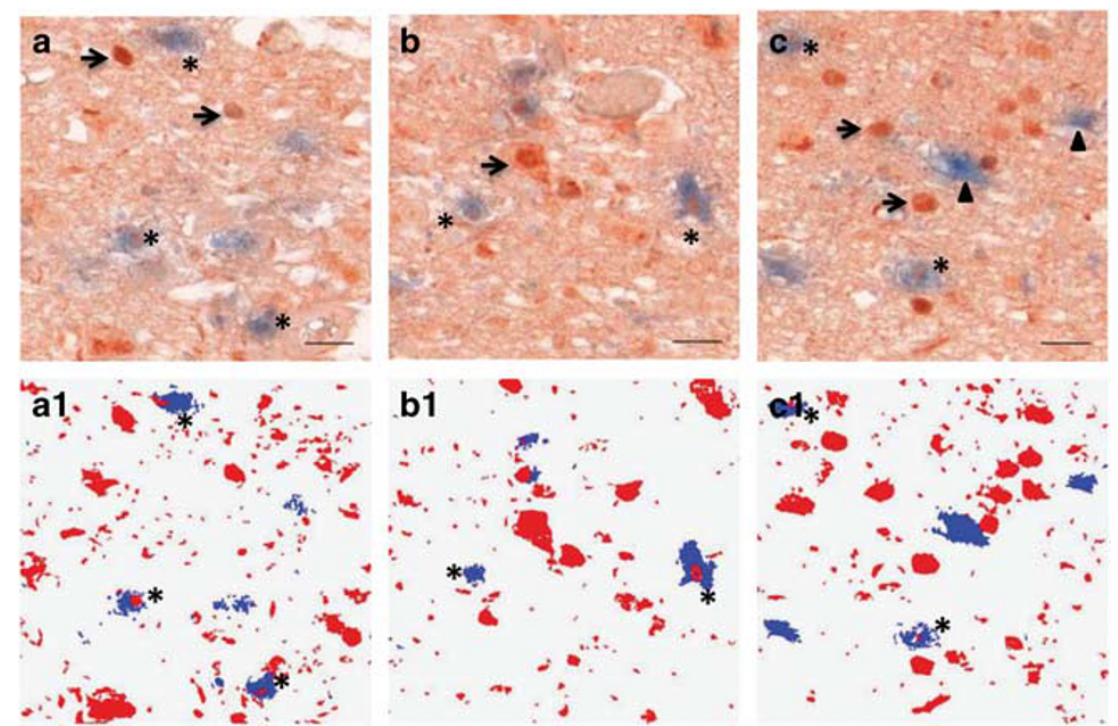

Figure 2 (a-c) Co-localization of androgen receptor (AR) and oxytocin (OXT) in the hypothalamic paraventricular nucleus (PVN). Asterisks indicate colocalization of AR (red) and OXT (blue), arrows indicate single AR-stained cells, and arrowheads indicate single OXT-stained cells. Bar $=25 \mu \mathrm{m}$. (a I-cl) the mask of AR (red) and OXT (blue) signals which had optical density values more than two times the background. The double-stained cells are indicated by asterisks.

a
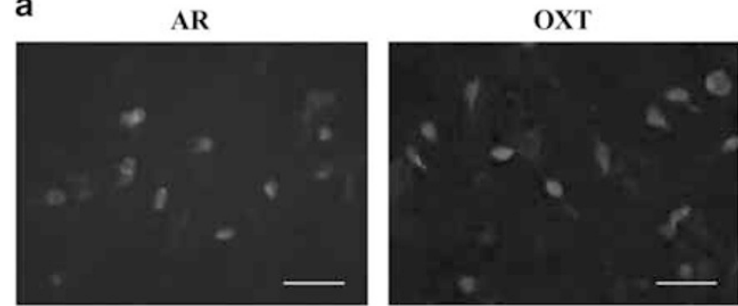

b

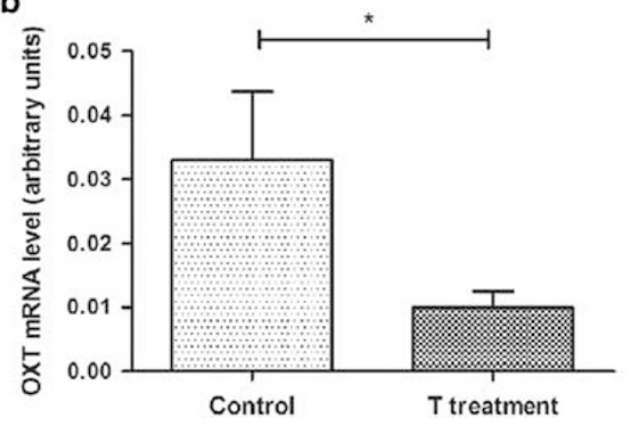

Figure 3 Endogenous expression of androgen receptor (AR) and oxytocin (OXT) in SK-N-SH cells, and the comparison of OXT-mRNA levels between testosterone treated cells and controls. (a) Immunofluorescence staining for the endogenous expression of AR (a, left) and OXT (a, right) in SK-N-SH cells. Bar $=50 \mu \mathrm{m}$. (b) Comparison of OXT-mRNA levels in SK-N-SH cells with adding in the control group the estrogen receptor antagonist ICI I 82780 to the medium and adding ICI I 82780 followed by testosterone administration for $24 \mathrm{~h}$ to the medium of the experimental group. Data are presented as mean \pm SEM from three independent experiments. ${ }^{*} p=0.05$. $T$ treatment: Testosterone treatment.

among chronically depressed individuals (Purba et al, 1996; Turan et al, 2013) thus seem puzzling. However, it has been proposed that higher OXT expression in depression might reflect a compensatory role: it may alleviate depressive symptoms and anxiety (McQuaid et al, 2014). Indeed, OXT and $\mathrm{CRH}$ have opposing effects: $\mathrm{CRH}$ stimulates adrenocorticotropic hormone and cortisol release, while OXT inhibits corticotropic function in humans (Legros, 2001). Alternatively, OXT may increase the salience of social stimuli, irrespective of whether these stimuli are positive or negative. In this wayalso depending on OXT receptor-related polymorphismsOXT could be associated with greater sensitivity and susceptibility to disturbed social relations, depressive symptoms and suicidal ideation (McQuaid et al, 2016).

The human OXT gene expression was found to be up-regulated by estrogens via ER (Richard and Zingg, 1990). In addition, also the androgen metabolite, ie $5 \alpha$-androstane, $3 \beta$, $17 \beta$-diol ( $3 \beta$-diol), was found to be capable to up-regulate OXT expression via $\mathrm{ER} \beta$ by combining it with a hormoneresponsive element in the rat OXT gene promoter (Hiroi et al, 2013; Sharma et al, 2012). This is why we used the ER antagonist ICI 182780 in our experiments. With the neuroblastoma cell line SK-N-SH, which had endogenous $\mathrm{ER}, \mathrm{AR}$ and OXT expression, we observed now for the first time that testosterone induced decreased OXT-mRNA expression. It is of importance to note that changes (either larger or smaller) in AR expression in mood disorders do not necessarily have an a priori correlation to the changes in OXT, since there are numerous other factors, such as many neurotransmitters and ER, involved in the regulation of OXT expression. However, the hypothesis that AR might play a role in the higher OXT-immunoreactivity in mood disorders is not only supported by our molecular work that indicates a direct regulating role of AR on OXT gene expression, but also by the lower testosterone levels observed in elderly depressed males (Rizvi et al, 2010) and females (Yasui et al, 2012).

One of the inherent potential confounding factors in any postmortem study is medication. However, we do not think that our main conclusions are confounded by antidepressants. Some previous studies have shown that there were no obvious changes in plasma OXT concentration during selective serotonin reuptake inhibitor (SSRI) or electroconvulsive therapy treatment of depressed patients (Keating et al, 2013; Ozsoy et al, 2009) or during SSRI treatment of 
a

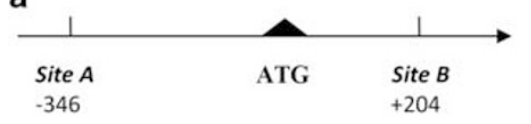

ARE motif NGNACANNNTGTNC

Site A AGgtactgGGaGGCT

Site B GAGAACTCCAGGAGC

C

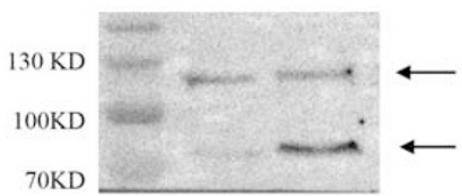

b
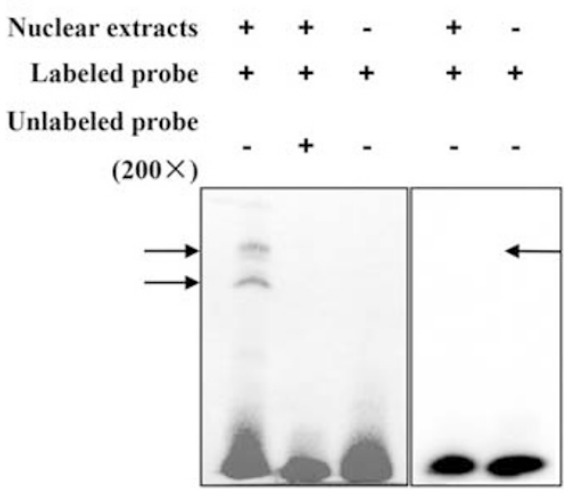

site A

site B

Figure 4 Electrophoretic mobility shift assay (EMSA) of two predicted androgen responsive elements (AREs). (a) Comparison of ARE sites A and site $B$ in the human oxytocin (OXT) gene promoter with a consensus ARE. Site $A$ is located at -346 to -332 , while site $B$ is located at +204 to +218 , relative to the start site ATG. Nucleotides of site A and site B are shown respectively. $N=$ any nucleotide. (b) EMSA performed with androgen receptor (AR)-containing nuclear extracts and potential ARE sequences site A and site B. Site A strongly bound to nuclear extracts from PSV-AR-transfected 293T cells, as is evident from the band shift indicated by arrows. Bands completely disappeared when nuclear extracts were incubated with a 200-fold excess of non-labeled probe. The same holds true when nuclear extracts were omitted from the reaction mixture. (c) Western blot detection of AR variants. $293 T$ cells were transfected with human AR expressing plasmid PSV-AR and detected by western blot. AR variants were indicated by two arrows between 70 KD and I 30 KD, which were consistent with the literature (Saatcioglu, 20II; Wilson and McPhaul, 1996).

a

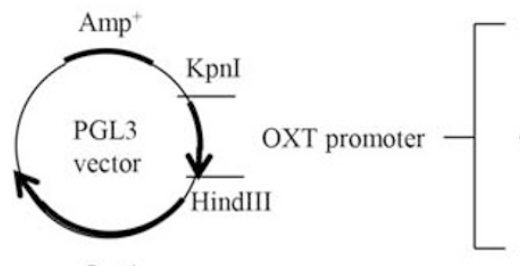

Luc $^{+}$
OXT promoter

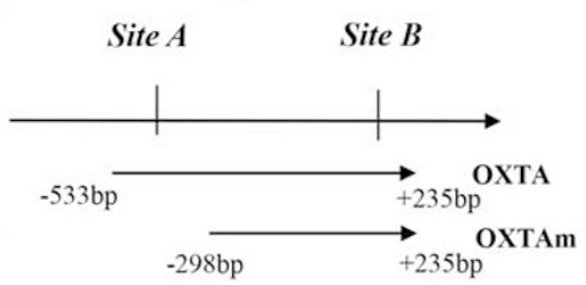

c

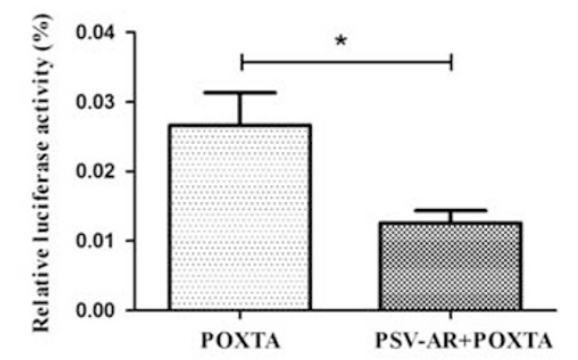

b

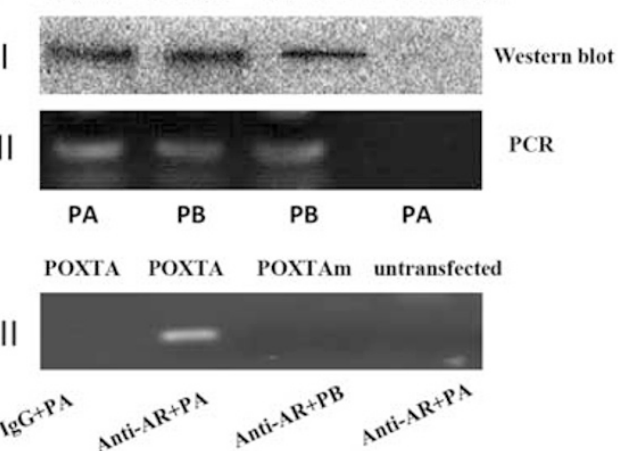

Figure 5 Inhibitory effect of testosterone on oxytocin (OXT) gene expression via androgen responsive element (ARE). (a) Diagram of the two fragments from the human OXT promoter sequence used in construction of the reporter plasmids. Full-length OXT promoter (OXTA), spanning both ARE sites, A and B, from - 533 bp to 235 bp, was cloned into the PGL3 luciferase reporter basic vector. The OXTAm fragment is shorter and lacks site A. (b) An interaction assay was performed on 293FT cells co-transfected with PSV-androgen receptor (AR) and one of the OXT promoter plasmids (POXTA or POXTAm). Panel I: Western blot analyses were performed to determine the presence of AR after co-transfection, compared with the untransfected 293FT cells. Panel II: PCR amplification of ARE site A and site B in nucleus sonication products by Primer A (PA) and Primer B (PB). Panel III: Immunoprecipitated complexes were detected by PCR analysis. Only 293 FT cells co-transfected with POXTA and incubated with anti-AR antibody demonstrated PCR product when Primer A was used. However, cells co-transfected with POXTAm and incubated with anti-AR antibody did not show the PCR products when Primer B was used. In addition, neither untransfected cells treated with anti-AR antibody nor cells co-transfected with POXTA and incubated with IgG demonstrated PCR products when Primer A was used. (c) Luciferase activity in 293FT cells, which were co-transfected with human OXT promoter plasmids POXTA with or without plasmid contained AR (PSV-AR), followed by testosterone treatment. Note the significantly decreased luciferase activity in 293FT cells transfected with PSV$A R$, pointing to an inhibitory role of testosterone via AR on OXT gene expression. Results are expressed as mean luciferase activity $\pm S E M$ from three independent experiments. $* 0=0.0126$. 
obsessive-compulsive disorder patients (Humble et al, 2013). In addition, Haloperidol, which had been given to one mood disorder patient and three controls in the present study, was found not to influence OXT release in rat (Uvnäsmoberg et al, 1992). Moreover, although Lithium (You et al, 2001) and Valproate (Štefánik et al, 2015) are reported to increase OXT release in rats and to increase OXT mRNA expression in rat hypothalamic PVN and SON, the IOD of OXTimmunoreactivity of the mood disorder patients who had been administered these compounds (IOD: 24.41, 27.39, and 18.06) were fully within the range of the other mood disorder patients (from 16.06 to 40.91, mean value 24.02). Furthermore, a benzodiazepine, such as chlordiazepoxide, was shown to inhibit OXT release in response to noxious stimuli (Yagi and Onaka, 1996), and chronic morphine treatment in rat inhibited OXT synthesis (Morris et al, 2010; You et al, 2001). In our study, more mood disorder patients $(n=9)$ than control subjects $(n=3)$ were given diazepines, and more mood disorder patients $(n=9)$ than control subjects $(n=3)$ were exposed to morphine. Had these compounds interfered with our measurements, then this would have led to an underestimation of the increased OXT-immunoreactivity levels observed in mood disorder patients.

We subsequently identified a specific ARE site in the human OXT promoter, which can, in principle, be located either upstream or downstream of the transcription start site of androgen responsive genes (Saatcioglu, 2011). We focused on one potential ARE site (named site A in the present study), near the start site ATG, which is most likely involved in the regulation of $O X T$ gene expression. We observed, by means of EMSA, that this ARE (site A) does indeed bind to AR nuclear extracts, as evidenced by two separated bands and consistent with a previous report of two AR isoforms (Saatcioglu, 2011; Wilson and McPhaul, 1996). Human OXT promoter transcriptional activity showed a significant decrease following cotransfection with an AR-expression plasmid and testosterone treatment, which indicated that testosterone may suppress OXT expression. Evidence from a previous study indicates a clear interplay between AR and ER, which may be either competitive or antagonistic (Need et al, 2012). Therefore, the blocking of ER with ICI 182780 in our experiments might potentiate the inhibitory effect of AR on OXT expression in SK-N-SH cells. Our data offer the first evidence of a direct inhibiting effect of androgens on human OXT expression.

It is of interest to note that AR knock-out mice showed higher expression levels of OXT receptor (OXTR) in the hypothalamus than controls (Karlsson et al, 2016), which is consistent with our present findings. These findings together indicate that testosterone may have additive effects on oxytocinergic function. It should be noted, however, that there are also data indicating that androgens do not influence the expression of OXTR (Filippi et al, 2002). Whether OXTR may act as an additional potential target for androgen actions needs to be elucidated in the future.

In conjunction with data from others, androgens may thus participate in the regulation of OXT levels, either inhibiting directly via $\mathrm{AR}$, or indirectly stimulating $O X T$ expression through its metabolite via ER (see above), both in health and in disease. In conclusion, there is more hypothalamic PVN OXT expression in mood disorders and co-localization of AR in human OXT neurons. Our observation that OXT expression is regulated by a direct inhibitory effect of the
AR on OXT transcription may be the basis for the opposing effects of OXT and testosterone in psychological functions and neuropsychiatric disorders as proposed in the literature (Crespi, 2015).

\section{FUNDING AND DISCLOSURE}

Professor Ai-Min Bao was supported by the National Key Research and Development Program of China (2016YFC1 306701), the National Natural Science Foundation of China (91332102), and the Key Laboratory of Mental Disorder's Management of Zhejiang Province. Professor Dick Swaab was supported by Programme of Introducing Talents of Discipline to Universities of China (B13026). Dr Shao-Hua $\mathrm{Hu}$ was supported by the Key Laboratory of Mental Disorder's Management of Zhejiang Province. Miss Dan Dai was supported by the China Scholarship Council for State Scholarship Fund (grant number 201406320021). The authors declare no conflict of interest.

\section{ACKNOWLEDGMENTS}

We are grateful to the Netherlands Brain Bank (Director Dr Inge Huitinga) for providing human brain material and clinical details, to Professor Joost Verhaagen for critically reading the manuscript, to Dr F.W. van Leeuwen for providing the OXT antibody, to Dr J. Trapman for providing the plasmid PSV-AR and to Ms W.T.P. Verweij for her secretarial assistance.

\section{REFERENCES}

Bao A, Fischer D, Wu Y, Hol E, Balesar R, Unmehopa U et al (2006). A direct androgenic involvement in the expression of human corticotropin-releasing hormone. Mol Psychiatry 11: 567-576.

Baxter LC (2016). Appetite changes in depression. Am J Psychiatry 173: 317-318.

Bennett NC, Gardiner RA, Hooper JD, Johnson DW, Gobe GC (2010). Molecular cell biology of androgen receptor signalling. Int J Biochem Cell Biol 42: 813-827.

Bingaman EW, Baeckman LM, Yracheta JM, Handa RJ, Gray TS (1994). Localization of androgen receptor within peptidergic neurons of the rat forebrain. Brain Res Bull 35: 379-382.

Carson DS, Guastella AJ, Taylor ER, McGregor IS (2013). A brief history of oxytocin and its role in modulating psychostimulant effects. J Psychopharmacol 27: 231-247.

Cochran D, Fallon D, Hill M, Frazier JA (2013). The role of oxytocin in psychiatric disorders: a review of biological and therapeutic research findings. Harv Rev Psychiatry 21: 219.

Crespi BJ (2015). Oxytocin, testosterone, and human social cognition. Biol Rev 91: 390-408.

Fernándezguasti A, Kruijver FP, Fodor M, Swaab DF (2000). Sex differences in the distribution of androgen receptors in the human hypothalamus. J Comp Neurol 425: 422-435.

Filippi S, Luconi M, Granchi S, Vignozzi L, Bettuzzi S, Tozzi P et al (2002). Estrogens, but not androgens, regulate expression and functional activity of oxytocin receptor in rabbit epididymis. Endocrinology 143: 4271-4280.

Fronczek R, Lammers GJ, Balesar R, Unmehopa UA, Swaab DF (2005). The number of hypothalamic hypocretin (orexin) neurons is not affected in Prader-Willi syndrome. J Clin Endocrinol Metab 90: 5466-5470.

Gao SF, Qi XR, Zhao J, Balesar R, Bao AM, Swaab DF (2013). Decreased NOS1 expression in the anterior cingulate cortex in depression. Cerebr Cortex 23: 2956-2964. 
Heinrichs M, Domes G (2008). Neuropeptides and social behaviour: effects of oxytocin and vasopressin in humans. Prog Brain Res 170: 337-350.

Hiroi R, Lacagnina AF, Hinds LR, Carbone DG, Uht RM, Handa RJ (2013). The androgen metabolite, $5 \alpha$-androstane- $3 \beta, 17 \beta$-diol ( $3 \beta$ diol), activates the oxytocin promoter through an estrogen receptor- $\beta$ pathway. Endocrinology 154: 1802-1812.

Hofmann SG, Fang A, Brager DN (2015). Effect of intranasal oxytocin administration on psychiatric symptoms: a meta-analysis of placebo-controlled studies. Psychiatry Res 228: 708-714.

Humble MB, Uvnäs-Moberg K, Engström I, Bejerot S (2013). Plasma oxytocin changes and anti-obsessive response during serotonin reuptake inhibitor treatment: a placebo controlled study. BMC Psychiatry 13: 1.

Karlsson SA, Studer E, Kettunen P, Westberg L (2016). Neural androgen receptors modulate gene expression and social recognition but not social investigation. Front Behav Neurosci 10: 1-13.

Keating C, Dawood T, Barton DA, Lambert GW, Tilbrook AJ (2013). Effects of selective serotonin reuptake inhibitor treatment on plasma oxytocin and cortisol in major depressive disorder. BMC Psychiatry 13: 124.

Knobloch HS, Charlet A, Hoffmann Lena C, Eliava M, Khrulev S, Cetin Ali $\mathrm{H}$ et al (2012). Evoked axonal oxytocin release in the central amygdala attenuates fear response. Neuron 73: 553-566.

Lawson EA, Holsen LM, Santin M, Meenaghan E, Eddy KT, Becker $\mathrm{AE}$ et al (2012). Oxytocin secretion is associated with severity of disordered eating psychopathology and insular cortex hypoactivation in anorexia nervosa. J Clin Endocrinol Metab 97: E1898-E1908.

Legros J-J (2001). Inhibitory effect of oxytocin on corticotrope function in humans: are vasopressin and oxytocin ying-yang neurohormones? Psychoneuroendocrinology 26: 649-655.

Leng G, Ludwig M (2016). Intranasal oxytocin: myths and delusions. Biol Psychiatry 79: 243-250.

McQuaid RJ, McInnis OA, Abizaid A, Anisman H (2014). Making room for oxytocin in understanding depression. Neurosci Biobehav Rev 45: 305-322.

McQuaid RJ, McInnis OA, Matheson K, Anisman H (2016). Oxytocin and social sensitivity: gene polymorphisms in relation to depressive symptoms and suicidal ideation. Front Hum Neurosci 10.

Morris MS, Domino EF, Domino SE (2010). Opioid modulation of oxytocin release. J Clin Pharmacol 50: 1112-1117.

Murakami G, Hunter RG, Fontaine C, Ribeiro A, Pfaff D (2011). Relationships among estrogen receptor, oxytocin and vasopressin gene expression and social interaction in male mice. Eur $J$ Neurosci 34: 469-477.

Need EF, Selth LA, Harris TJ, Birrell SN, Tilley WD, Buchanan G (2012). Research resource: interplay between the genomic and transcriptional networks of androgen receptor and estrogen receptor $\alpha$ in luminal breast cancer cells. Mol Endocrinol 26: 1941-1952.

Ozsoy S, Esel E, Kula M (2009). Serum oxytocin levels in patients with depression and the effects of gender and antidepressant treatment. Psychiatry Res 169: 249-252.

Purba JS, Hoogendijk WJ, Hofman MA, Swaab DF (1996). Increased number of vasopressin-and oxytocin-expressing neurons in the paraventricular nucleus of the hypothalamus in depression. Arch Gen Psychiatry 53: 137-143.

Quirin M, Kuhl J, Düsing R (2011). Oxytocin buffers cortisol responses to stress in individuals with impaired emotion regulation abilities. Psychoneuroendocrinology 36: 898-904.
Richard SP, Zingg H (1990). The human oxytocin gene promoter is regulated by estrogens. J Biol Chem 265: 6098-6103.

Rizvi SJ, Kennedy SH, Ravindran LN, Giacobbe P, Eisfeld BS, Mancini D et al (2010). The relationship between testosterone and sexual function in depressed and healthy men. J Sexual Med 7: 816-825.

Saatcioglu F. Androgen Action: Methods and Protocols. Humana Press: Hatfield, UK, 2011.

Sharma D, Handa RJ, Uht RM (2012). The ER $\beta$ ligand $5 \alpha$ androstane, $3 \beta, 17 \beta$-diol ( $3 \beta$-diol) regulates hypothalamic oxytocin (Oxt) gene expression. Endocrinology 153: 2353-2361.

Smets LA, Loesberg C, Janssen M, Metwally EA, Huiskamp R (1989). Active uptake and extravesicular storage of m-iodobenzylguanidine in human neuroblastoma SK-N-SH cells. Cancer Res 49: 2941-2944.

Smith AS, Ågmo A, Birnie AK, French JA (2010). Manipulation of the oxytocin system alters social behavior and attraction in pairbonding primates, Callithrix penicillata. Hormones Behav 57: 255-262.

Štefánik P, Olexová L, Kršková L (2015). Increased sociability and gene expression of oxytocin and its receptor in the brains of rats affected prenatally by valproic acid. Pharmacol Biochem Behav 131: $42-50$

Turan T, Uysal C, Asdemir A, Kılıç E (2013). May oxytocin be a trait marker for bipolar disorder? Psychoneuroendocrinology 38: 2890-2896.

Uvnäsmoberg K, Alster P, Svensson TH (1992). Amperozide and clozapine but not haloperidol or raclopride increase the secretion of oxytocin in rats. Psychopharmacology 109: 473-476.

van de Nes JA, Kamphorst W, Ravid R, Swaab DF (1998). Comparison of beta-protein/A4 deposits and Alz-50-stained cytoskeletal changes in the hypothalamus and adjoining areas of Alzheimer's disease patients: amorphic plaques and cytoskeletal changes occur independently. Acta Neuropathol 96: 129-138.

Wade TD, Bulik CM, Neale M, Kendler KS (2000). Anorexia nervosa and major depression: shared genetic and environmental risk factors. Am J Psychiatry 157: 469-471.

Wang S, Kamphuis W, Huitinga I, Zhou J, Swaab D (2008). Gene expression analysis in the human hypothalamus in depression by laser microdissection and real-time PCR: the presence of multiple receptor imbalances. Mol Psychiatry 13: 786-799.

Watson GS (1982). Circular statistics in biology. Technometrics 24: 336-336.

Watts AG (2005). Glucocorticoid regulation of peptide genes in neuroendocrine $\mathrm{CRH}$ neurons: a complexity beyond negative feedback. Front Neuroendocrinol 26: 109-130.

Wilson CM, McPhaul MJ (1996). A and B forms of the androgen receptor are expressed in a variety of human tissues. Mol Cell Endocrinol 120: 51-57.

Yagi K, Onaka T (1996). A benzodiazepine, chlordiazepoxide, blocks vasopressin and oxytocin release after footshocks but not osmotic stimulus in the rat. Neurosci Lett 203: 49-52.

Yasui T, Matsui S, Tani A, Kunimi K, Yamamoto S, Irahara M (2012). Androgen in postmenopausal women. J Med Investig 59: $12-27$.

You ZD, Li JH, Song CY, Lu CL, He C (2001). Oxytocin mediates the inhibitory action of acute lithium on the morphine dependence in rats. Neurosci Res 41: 143-150.

Zhou L, Blaustein JD, De Vries GJ (1994). Distribution of androgen receptor immunoreactivity in vasopressin- and oxytocinimmunoreactive neurons in the male rat brain. Endocrinology 134: $2622-2627$.

Supplementary Information accompanies the paper on the Neuropsychopharmacology website (http://www.nature.com/npp) 